\title{
Estimating Prevalence Rates of Women Diagnosed with Breast Cancer in Kilifi County
}

\section{Leonard Kiti Alii*}

Department of Mathematics and Statistics, Pwani University Kilifi, Kenya

\begin{abstract}
In this paper, analysis was done for patients diagnosed of cancer from the Kilifi county hospital. The presence or absence of breast cancer had been done by the medical personnel and data documented. The objective was to determine the cancer prevalence rates of in the county. Data was obtained from survey questions and diagnosis by the medical personnel within the observation and follow up period of the patients. Data was also obtained for patients that had undergone testing to ascertain the type of tumor they had. Chi-square tests were carried out to check whether there was association between cancer and the smoking and between cancer and alcohol intake. The test show there was no association between Cancer and smoking $\left(\chi^{2}=0.70938, d f=2, p v a l u e=0.7014\right)$. Similarly a chi-square test showed no association between breast cancer and alcohol intake $\left(\chi^{2}=0.42101, d f=2, p v a l u e=0.8102\right)$. A logistic regression was fit to adjust for confounding. The table below shows the results after fitting this model. The results confirm that smoking and alcohol intake was not associated with breast cancer.
\end{abstract}

Keywords: Breast cancer; Logistic regression; Prevalence; Chisquare; Association

\section{Background Information}

According to the Kenya Cancer Statistics and National Strategies (2013), Cancer causes more deaths than HIV, TB and Malaria combined. $70 \%$ of the global Cancer burden is in low and middle income countries like Kenya. $30 \%$ of cancers are treatable with prolonged survival if detected early; $30 \%$ of cancer patients can be provided with adequate symptom management and palliative care [1-5]. Cancer is the $3^{\text {rd }}$ highest cause of morbidity in Kenya [7\% of deaths per year], after infectious diseases and cardiovascular diseases. Its estimated that there are 39,000 new cases of Cancer each year in Kenya with more than 27,000 deaths per year. $60 \%$ of Kenyans affected by Cancer are younger than 70 years old. Leading Cancers are Women estimated at 34 per 100,000 for Breast cancer and estimated at 25 per 100,000 for Cervical cancer [6]. In Men Prostate cancer accounts for 17 per 100,000 and 9 per 100,000 for Esophageal cancer.70-80\% of cancer cases are diagnosed in late stages due to Lack of awareness, Inadequate diagnostic facilities, Lack of treatment facilities, High cost of treatment and High poverty Index. While there has been substantial research published on risk factors and prognostic factors for breast cancer in general, research specific to Kilifi is sparse. Further, the association between breast cancer survival and socio-demographic and pathologic factors has been widely studied but the majority of these studies are from developed countries. Most of these studies have not focused on survival and prognostic factors $[7,8]$. To our knowledge, no study has investigated breast cancer survival in this region or explored the relationships between the survival and socio-demographic and pathological factors. This paper gives results of a study which fills this. Ethical approval for this study will be obtained from the Research Ethics Committee of Pwani University.

\section{Kilifi county profile}

Kilifi County is located in the former Coast Province of Kenya, about $420 \mathrm{~km}$ south-east of Nairobi and $60 \mathrm{~km}$ north of Mombasa. It covers an area of 12,609.74 square kilometers and is home to 1,320,646 people (male - $48 \%$ and female - 52\%). Kilifi County has some of the poorest social economic indicators in the country with a poverty rate of $72 \%$ and a relatively low literacy level which exposes a significant proportion of the population to various health risk factors. Kilifi County has a total of 256 health facilities 108 being public health facilities and the other 148 being private facilities

\section{Justification}

According to the Kenya Cancer Statistics and National statistics Cancer is the $3^{\text {rd }}$ highest cause of morbidity in Kenya [7\% of deaths per year], after infectious diseases and cardiovascular diseases. Surveys carried out by the Malindi sub county hospital about 60 per cent of women in Kilifi County are not aware of the Breast Cancer. There is need to carry out a comprehensive study to understand the prevalence of breast Cancer of women diagnosed with Breast cancer and Stomach tumors in Kilifi county [9-12]. While there has been some awareness and forums for cancer screening, including cervical and breast cancer in some selected areas in Kilifi County, research regarding risk factors and prognostic factors for Breast cancer and growth of tumors in Kilifi County is sparse. Further, the association between Cancer and sociodemographic as well as pathologic factors has been scantly studied and the majority of these studies are from developed countries [13]. Apart from this study there is no evidence of research in the literature of research that has been done to determine prevalence of breast cancer and to explore the association between the survival and sociodemographic factors in Kilifi County.

\section{Methodology}

The data was obtained from the cancer registry in the County hospital, Kilifi County referral hospital. Follow-up started for those who had been diagnosed with a first primary invasive breast malignancy and had undergone breast surgery including axillary dissection [14].

*Corresponding author: Alii LK, Department of Mathematics and Statistics, Pwan University Kilifi, Kenya, Tel: 0722917294; E-mail: leonardalii@yahoo.com

Received October 23, 2017; Accepted November 24, 2017; Published November 27,2017

Citation: Alii LK (2017) Estimating Prevalence Rates of Women Diagnosed with Breast Cancer in Kilifi County. J Biom Biostat 8: 382. doi: 10.4172/21556180.1000382

Copyright: @ 2017 Alii LK. This is an open-access article distributed under the terms of the Creative Commons Attribution License, which permits unrestricted use, distribution, and reproduction in any medium, provided the original author and source are credited. 
The data obtained was used to compute the prevalence rates. Smoking status and alcohol intake of the patients was recorded. Chisquare tests and logistic regression were used to test for any association between smoking, alcohol intake and cancer. The results were represented interms of tables and graphs.

\section{The logistic regression model}

The logistic regression was used specifically because of its ability to control for confounding variables when modeling a categorical outcome variable. Logistic regression is a very popular analytical tool in medical studies. It's used to model probabilities using the properties of the sigmoid curve given by eqn. (1) below (Figure 1).

$$
P(Y)=\frac{1}{1+e^{-n}}
$$

Eqn. (1) above show how the sigmoid curve can be used to model the probability of occurrence of a disease since its range is always between 0 and 1 as illustrated in the figure below, when the independent variable varies from $-\infty$ to $\infty$,

From eqn. (1), it follows that when there is only one predictor variable $X_{1}$, the logistic regression equation from which the probability of $Y$ is predicted is given by

$$
P(Y)=\frac{1}{1+e^{-\left(\beta_{0}+\beta_{1} X\right)}}
$$

in which $\mathrm{P}(\mathrm{Y})$ is the probability of $Y$ occurring, $e$ is the base of natural logarithms, and the other coefficients form a linear combination in the independent variable $\mathrm{X}$, it is possible to extend this equation so as to include several predictors. When there are several predictors the equation becomes:

$$
P(Y)=\frac{1}{1+e^{-\left(\beta_{o}+\beta_{1} X_{1 i}+\ldots+\beta_{n} X_{n i}\right)}}
$$

Eqn. (3) illustrates how the logistic regression can control confounding and assess interaction very effectively when there are several confounders or the confounder is a continuous variable. Its most attractive advantage may be that the researcher can calculate an odds ratio and its confidence interval directly, so that the results can be

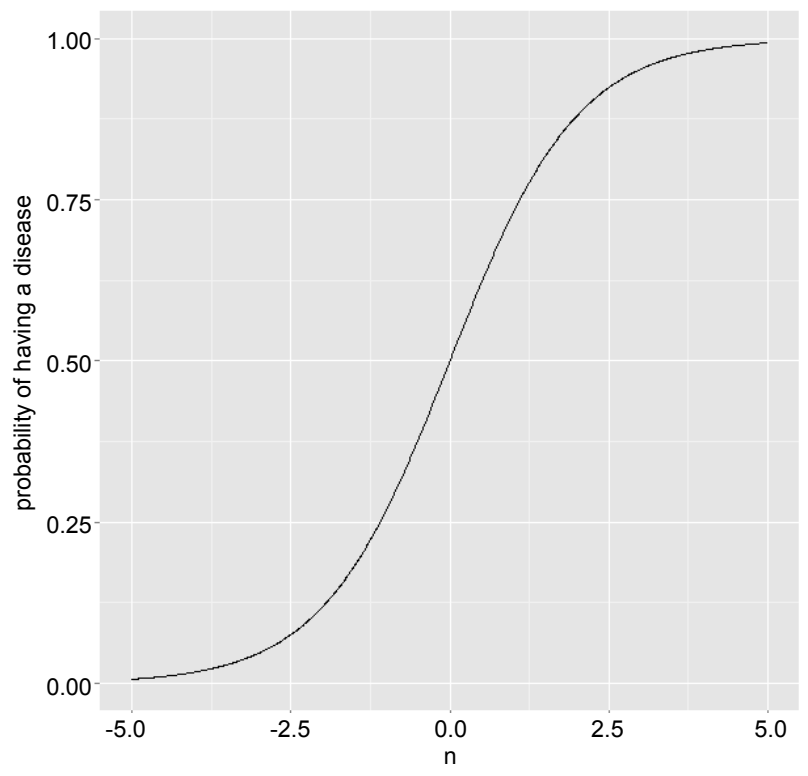

Figure 1: Graph shows Probability of having a disease. interpreted easily [15]. The probability of a given subject developing a disease during a fixed time interval can also be calculated. Logistic regression has also been extended to analyze data in which the dependent variable has more than two levels or the dependent variable is an ordinal variable. Effect of the socio-demographic and pathological factors options will be investigated using Cox regression. All analysis was conducted using R software.

\section{Discussions and Results}

This was the first study to evaluate breast cancer survival in Kilifi County and used a wide range of explanatory factors. The data obtained from the registry which was as from 2014-2016 had 30 tumour related cases, out of which 20 (66.67\%) were cervical cancer cases, 3(10\%) were breast cancer cases and $7(23.33 \%)$ had tumours that were unrelated to cancer [16-20]. According to the Kilifi county data from the population Census, the prevalence rates for Cervical Cancer was estimated at 15.4 per 100,000 women while Breast Cancer was estimated to be 2.3 per 100,000 women. These figures were relatively low compared to the national statistics which stand at 34 per 100,000 women for breast cancer and Breast cancer and 25 per 100,000 women for cervical cancer. The implication of this may be that many women may not know their status cancer status. A survey needs to be done to certain the levels of Cancer in the county.

The breast cancer stage of the patient was also noted. Of those who were cancerous $26.0 \%$ were at stage four, $43.5 \%$ were at stage three, $8.7 \%$ were at stage two and $17.4 \%$ were at stage one [21]. The age specific rates were calculated. The table below shows that most of the patients who were suffering from breast cancer were above 35 years. However, majority of the patients were above 60 years (Table 1 ).

A chi-square test was carried out to check whether there was association between cancer and the smoking and between cancer and alcohol intake (Figure 2). The test show there was no association between Cancer and smoking $\left(\chi^{2}=0.70938, d f=2\right.$, pvalue $\left.=0.7014\right)$. Similartly a chisquare test showed no association between breast cancer and alcohol intake $\left(\chi^{2}=0.42101, d f=2, p v a l u e=0.8102\right)$. A logistic regression was fit to adjust for confounding. The table below shows the results after fitting this model. The results confirm that smoking and alcohol intake was not associated with breast cancer (Table 2).

\section{Conclusion}

In this paper, analysis was done for patients diagnosed of breast cancer from the Kilifi county hospital. Prevalence rates calculated

\begin{tabular}{|c|c|c|c|c|}
\hline Age group & No of Women & Tumor & Cancerous & $\begin{array}{c}\text { Age specific prevalence } \\
\text { rate }\end{array}$ \\
\hline $15-19$ & 24654 & 0 & 0 & 0 \\
\hline $20-24$ & 22,300 & 1 & 0 & 0 \\
\hline $25-29$ & 18,147 & 0 & 0 & 0 \\
\hline $30-34$ & 15,020 & 0 & 0 & 0 \\
\hline $35-39$ & 10,642 & 1 & 1 & 12.4 \\
\hline $40-44$ & 8,189 & 1 & 1 & 28.2 \\
\hline $45-49$ & 7,102 & 3 & 2 & 44.5 \\
\hline $50-54$ & 6,748 & 5 & 3 & 46.5 \\
\hline $55-59$ & 4,299 & 2 & 2 & 129.9 \\
\hline $60-64$ & 3,848 & 6 & 5 & 70.5 \\
\hline $65-69$ & 2,836 & 3 & 2 & 237.9 \\
\hline $70-74$ & 2,102 & 6 & 5 & 0 \\
\hline $75-79$ & 1,440 & 0 & 0 & 88.2 \\
\hline $80+$ & 2,268 & 2 & 2 & \\
\hline
\end{tabular}

Table 1: Patients who were suffering from breast cancer were above 35 years. 


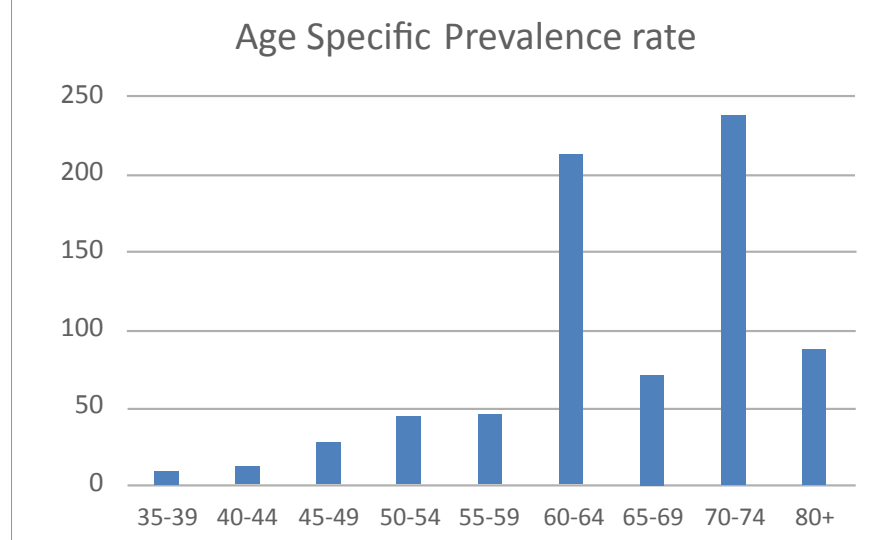

Figure 2: Graph shows age specific prevalence rate.

\begin{tabular}{|c|c|c|c|c|}
\hline Coefficient & Estimate & Std. Error & $\mathbf{z}$ value & $\operatorname{Pr}(>|\mathbf{z}|)$ \\
\hline Alcohol & 0.2843 & 1.6833 & 0.169 & 0.8659 \\
\hline Smoke & -0.3040 & 1.5375 & -0.198 & 0.8433 \\
\hline
\end{tabular}

Table 2: The results of a logistic regression.

showed that majority of the patients were above sixty years. This could explain that majority of the suffering from breast cancer in the early years could be missed cases and there is need for a survey to ascertain the true distribution of the cancer prevalence rates. Chi-square tests were carried out to check whether there was association between cancer and the smoking and between cancer and alcohol intake. The tests showed no association between Cancer and smoking. There was no association between breast cancer and alcohol intake.

\section{References}

1. Alice NP, Diane SM, Jasmine A, Stephen HT (2008) Mediating Factors in the Relationship between Income and Mammogram Use in Women. Journal of Women's Health 17: 1371-1378.

2. Bouchardy C, Verkooijen HM, Fioretta G (2006) Social Class is an Important and Independent Prognostic Factor for Breast Cancer Screening. Intl J Cancer 119: 1145-1151.

3. Bulaporn NS, Clark MJ (2008) Factors Influencing Breast Cancer screening among Older Thai. Hong Kong medical Journal 8: 334-340.

4. Calleb GO (2006) Breast Cancer Carcinoma at Coast Province General Hospital, Mombasa. East Africa Journal of Surgery 11: 10-14.
5. Cuthbertson SA, Goyder EC, Poole J (2009) Inequalities in Breast Cancer Diagnosis in the Trent region and Implications for the NHS Breast Screening Programme. J Public Health 31: 398-405.

6. Danaei G, Vander Hoorn S, Lopez AD, Murray CJ, Ezzati M (2005) Causes of cancer in the world: Comparative assessment of nine behavioral and environmental risk factors. The Lancet 366: 1784-1793.

7. Driscoll T, Nelson DI, Steenland K, Leigh J, Concha-Barrientos M, (2005) The global burden of diseases due to occupational carcinogens. American journal of Industrial Medicine 48: 419-431.

8. Farley J, Bray F, Pisan P, Parkin DM (2008) Cancer incidence, Mortality and Prevalence worldwide. Globocan IRAC 5.

9. Gulshan K, Lim JN, Hewison J, Atkin K, Horgan K (2007) Culture, Attitude and Knowledge about Breast Cancer and Preventive measures: a Qualitative Study of South Asian Breast Cancer Patients in the UK. Asian Pacific J Cancer Prev 12: 1619-1676.

10. Jayant K, Nene BM, Dinshaw KA, Budukh AM, Dale PS (1998) Survival from cervical cancer in Barchi registry, rural India. Cancer Survival in developing countries. IARC Scientific Publication, pp: 69-77.

11. Kenya National Bureau of Statistics (2010) Kenya Population and Housing Census Government press. Nairobi.

12. Maheswarab R, Pearson T, Jordan H (2006) Socio-Economic Deprivation, Travel distance, Location of service and Uptake of Breast Screening in North Derbyshire, UK. Journal of Epidemiology and Community Health 60: 208-212.

13. Matthers CD, Loncar D (2006) Projections of global mortality and burden of disease from 2002 to 2030. PloS Medicine 3: 2011-2030.

14. Miller AB (1984) The information explosion. The role of the epidemiologist. Cancer Forum 8: 67-75.

15. Moller H (2008) Breast Cancer Incidence, Care and Outcomes in Ethnic Groups in South East England. Royal Society of Medicine London.

16. Moser K, Patnick J, Beral V (2009) Inequalities in Reported Use of Breast Screening in Great Britain: Analysis of Cross Sectional Survey Data. Br Med J 338.

17. National Cancer Forum (2006) A Strategy for Cancer Control in Ireland.

18. Ponten J, Adami HO, Bergström R, Dillner J, Friberg L (1995) Strategies for control of cervical cancer. International journal of cancer 60: 1-26.

19. Turner PC, Sylla A, Gong YY, Diallo MS, Sutcliffe A (2005) Reduction in exposure to carcinogenic aflatoxins by postharvest intervention measures in West Africa: a community based intervention study. Lancet 10: 1950-1956.

20. United Nations Scientific Committee on the Effects of Atomic Radiation (2000) Sources and effects of ionizing radiation: sources. United Nations Publications 1.

21. World Health Organization (2002) National cancer control programmes: policies and managerial guidelines. World Health Organization. 